\title{
ON STOCHASTIC RECURSIVE EQUATIONS OF SUM AND MAX TYPE
}

\author{
LUDGER RÜSCHENDORF, ${ }^{*}$ Albert-Ludwigs-Universität Freiburg
}

\begin{abstract}
In this paper we consider stochastic recursive equations of sum type, $X \stackrel{\mathrm{D}}{=} \sum_{i=1}^{K} A_{i} X_{i}+b$, and of max type, $X \stackrel{\mathrm{D}}{=} \max \left\{A_{i} X_{i}+b_{i}: 1 \leq i \leq k\right\}$, where $A_{i}, b_{i}$, and $b$ are random, $\left(X_{i}\right)$ are independent, identically distributed copies of $X$, and ' $\stackrel{\text { D, }}{=}$ denotes equality in distribution. Equations of these types typically characterize limits in the probabilistic analysis of algorithms, in combinatorial optimization problems, and in many other problems having a recursive structure. We develop some new contraction properties of minimal $L_{s}$-metrics which allow us to establish general existence and uniqueness results for solutions without imposing any moment conditions. As an application we obtain a one-to-one relationship between the set of solutions to the homogeneous equation and the set of solutions to the inhomogeneous equation, for sum- and max-type equations. We also give a stochastic interpretation of a recent transfer principle of Rösler from nonnegative solutions of sum type to those of max type, by means of random scaled Weibull distributions.
\end{abstract}

Keywords: Additive recursive equation; recursive algorithm; contraction method

2000 Mathematics Subject Classification: Primary 60E05; 68W40

Secondary $60 \mathrm{H} 30$

\section{Introduction}

Stochastic recursive equations of sum and max type arise in a great variety of problems with a recursive stochastic component, as in the probabilistic analysis of algorithms or in combinatorial optimization problems. For a list of examples in these areas, see the recent survey [1] on max-recursive equations, and see [19] for additive equations. In particular, the limiting distribution of parameters of algorithms of divide-and-conquer type leads typically, for additive parameters such as path length or insertion depth in random trees, to equations of sum type, while parameters such as worst-case behaviour, height of random trees, and others lead typically to equations of max type. The contraction method is an effective tool for proving limit theorems and existence and uniqueness results for recursive algorithms and, in particular, recursive equations. The method was introduced for the analysis of the Quicksort algorithm in [25] and then independently developed further in [26] and [24] (which was submitted prior to [26]). It was then used and extended to the analysis of a large variety of algorithms in a series of papers; see, in particular, [19], [20], [21], and [27], which give general and easy-to-apply conditions for convergence results. The contraction method has also been successfully applied to some nonlinear stochastic equations, e.g. for the analysis of iterated function systems, random fractal measures, and fractal stochastic processes (see [13], [14], [15], and [24]).

There is an extensive literature on the characterization and existence of solutions to additive equations of sum type (as for branching type processes) and quite general existence results

Received 20 April 2005; revision received 28 June 2006.

* Postal address: Mathematisches Institut, Albert-Ludwigs-Universität Freiburg, Eckerstr. 1, 79104 Freiburg, Germany.

Email address: ruschen@stochastik.uni-freiburg.de 
are known in the homogeneous nonnegative case (see [3], [4], [5], [8], [12], [17], and [18]). Contraction arguments based on suitable probability metrics for this problem were given in [6], [24], and [26].

In particular, the minimal $L_{s}$-metric $\ell_{s}$ and the Zolotarev metric $\zeta_{s}$ have been applied to stochastic equations. For sum recursions, the metrics $\ell_{2}$ and $\zeta_{s}$ are particularly well suited. They yield good contraction factors for the distributional operator $T$ on the set, $M_{s}$, of distributions with finite $s$ th moments:

$$
T: M_{s} \rightarrow M_{s}, \quad T X \stackrel{\mathrm{D}}{=} \sum_{i=1}^{K} A_{i} X_{i}+b .
$$

Here $\left(X_{i}\right)$ are independent, identically distributed (i.i.d.) copies of $X,\left(A_{i}, b\right)_{1 \leq i \leq K}$ are independent of $\left(X_{i}\right)$, and ' $=$, denotes equality in distribution. We assume throughout the paper that $K<\infty$, even though most of our discussion can be extended directly to the case where $K=\infty$ or to the case where $K$ is random. We obtain

$$
\ell_{2}^{2}(T X, T Y) \leq \mathrm{E}\left(\sum_{i=1}^{K} A_{i}^{2}\right) \ell_{2}^{2}(X, Y),
$$

if $\mathrm{E} X=\mathrm{E} Y$, and, for all $s>0$,

$$
\zeta_{s}(T X, T Y) \leq \mathrm{E} \sum_{i=1}^{K}\left|A_{i}\right|^{s} \zeta_{s}(X, Y)
$$

see [23], [24], and [26]. For $0<s \leq 1, \ell_{s}$ has the same good contraction factor, E $\sum_{i=1}^{K}\left|A_{i}\right|^{s}$, as the $\zeta_{s}$-metric, but for $1<s<2$ we obtain only

$$
\ell_{s}(T X, T Y) \leq K_{s}\left(\mathrm{E} \sum_{i=1}^{K}\left|A_{i}\right|^{s}\right)^{1 / s} L_{s}(X, Y),
$$

for any coupling $(X, Y)$ with $\mathrm{E}(Y-X)=0$, where $K_{S}>1$ is a constant; see [23]. Inequality (1.3) is based on Woyczynski's inequality. It is valid in general Banach spaces under a type condition. For real random variables, the type is two and $K_{s}$ can be taken to be $K_{s}=18 s^{3 / 2}(s-1)^{1 / 2}$ for $1<s \leq 2$. In fact, in the real case Vatutin and Topchiı [30] and Alsmeyer and Rösler [2] established this inequality for $K=2$.

For max-type recursions it has been established in [21], [23], and [24] that the minimal $L_{s}$-metric $\ell_{s}$ is well suited even though it is not an ideal metric in the sense of Zolotarev. For the max operator, such that

$$
T X \stackrel{\mathrm{D}}{=} \bigvee_{i=1}^{K}\left(A_{i} X_{i}+b_{i}\right)
$$

where $\left(X_{i}\right)$ are again i.i.d. copies of $X$ independent of $\left(A_{i}, b_{i}\right)_{1 \leq i \leq K}$, and ' $\bigvee$ ' denotes the maximum, for any $s>0$ we obtain

$$
\ell_{S}(T X, T Y) \leq\left(\mathrm{E} \sum_{i=1}^{K}\left|A_{i}\right|^{s}\right)^{1 / s \wedge 1} \ell_{S}(X, Y)
$$


The contraction properties in (1.2)-(1.5) can be extended to random $K$ or to $K=\infty$ as well as to Banach spaces, but in this paper we restrict to the case of distributions and random variables in $\mathbb{R}^{1}$. In the following, we will freely use random variables or their distributions as arguments of the metrics.

For the application of contraction arguments to the problem of existence and characterization of solutions in the sum case, it is important also to be able to apply the $\ell_{s}$-metrics in the domain $1 \leq s \leq 2$, since, compared with the Zolotarev metric $\zeta_{s}$, they allow us much more easily to obtain upper estimates for the sum-recursive equation in (1.1). In Section 2 we prove that, in spite of the poor contraction factor $K_{s}$ in (1.3), we can obtain existence and uniqueness results for sum recursions with respect to $\ell_{s}$ for any $s, 1<s \leq 2$, under the natural contraction condition $\eta_{s}=\mathrm{E} \sum_{i=1}^{K}\left|A_{i}\right|^{s}<1$. The proof of this result uses a coupling construction based on weighted branching trees. We then extend the existence results without using any moment conditions on the solutions. To do so we introduce a new variant of the minimal $L_{s}$-metric, denoted $\ell_{s}^{0}$, which allows us to apply contraction arguments without imposing moment conditions. This extension of the applicability of $\ell_{s}$-metrics to the analysis of sum equations is the main contribution of this paper.

As a consequence of these developments we obtain an interesting equivalence theorem which establishes a one-to-one relationship between the sets of all solutions to homogeneous and, respectively, inhomogeneous additive recursive equations. For max-recursive sequences, the minimal $L_{S}$-metrics $\ell_{S}$ have recently been shown, in [21], to be ideally suited to obtaining existence and stability results. In Section 4 we establish the corresponding one-to-one relationship between homogeneous and inhomogeneous equations for max-recursive sequences. The basic structure of the arguments is the same as in the sum case. However, there are specific differences. While the $\ell_{s}$-metrics can be directly applied in the max case without any restriction, for $s>0$, they have to be considered in combination with weighted branching processes in the range $1 \leq s \leq 2$ in the sum case in $M_{1}$. Furthermore, for the general existence result in $M=M^{1}\left(\mathbb{R}^{1}, \mathcal{B}^{1}\right)$, the class of all probability distributions on $\left(\mathbb{R}^{1}, \mathscr{B}^{1}\right)$, we finally need to introduce the modified metrics $\ell_{s}^{0}$ (see Theorem 2.3). These differences prevent us from combining these results into a single master theorem.

We also give an analogue of Guivarc'h's transformation method for sum recursions (see [11]) in the case of max-recursive equations. This principle allows us to transfer nonnegative solutions to additive stochastic equations to max-recursive equations. A central role in this transformation is played by the Weibull distribution, and the solution set constructed in this way can be seen as a set of random scaled Weibull distributions. In operator language, this transfer was noted recently in [28].

Limits of max-recursive sequences constitute a basic source of max-recursive equations. We end the paper with an application of the recent limit theorem for max-recursive algorithms in [21] to the limit for the worst case of FIND, which is characterized by a max-recursive stochastic equation.

\section{Additive recursive equations: analysis using $\ell_{s}$-metrics}

For probability measures $\mu, v \in M=M^{1}\left(\mathbb{R}^{1}, \mathscr{B}^{1}\right)$, for $s>0$ we denote by $\ell_{s}(\mu, v)$ the minimal $L_{s}$-metric

$$
\ell_{s}(\mu, \nu)=\inf \left\{\left(\mathrm{E}|X-Y|^{s}\right)^{1 / s \wedge 1}: X \stackrel{\mathrm{D}}{=} \mu, Y \stackrel{\mathrm{D}}{=} v\right\} .
$$

Also, we synonymously use the notation $\ell_{s}(X, Y)$ or $\ell_{s}(X, \mu)$ for the distance of the corresponding distributions. While $\ell_{s}(\mu, v)$ in (2.1) is defined for all $\mu, v \in M$, it is not always 
finite. For $\mu \in M$, we denote by $M_{s}(\mu)$ the set of all $v \in M$ for which $\ell_{s}(\mu, v)$ is finite:

$$
M_{S}(\mu):=\left\{v \in M: \ell_{s}(\mu, v)<\infty\right\} .
$$

For any $\mu \in M_{s}$, the class of all probability measures with finite $s$ th moments, we have $M_{s}(\mu)=M_{s}$. For $s \geq 1$, we will additionally have to consider subsets of $M_{s}(\mu)$ in which the first moment is fixed to have the value $c$ :

$$
M_{S}(\mu, c)=\left\{v \in M_{1}: \ell_{s}(\mu, v)<\infty, \int x \mathrm{~d} v(x)=c\right\} .
$$

Let $T$ denote the operator, on the set of probability measures corresponding to (1.1), such that $T X \stackrel{\mathrm{D}}{=} \sum_{i=1}^{K} A_{i} X_{i}+b$. The $\ell_{s}$-metrics have the following contraction properties with respect to the operator $T$.

Lemma 2.1. Let $\mu_{0} \in M$ and $\mu, v \in M_{S}\left(\mu_{0}\right)$. Then,

(a) for any $s>0$, with $r:=\min (s, 1)$ we have

$$
\ell_{s}(T \mu, T \nu) \leq \sum_{i=1}^{K} \mathrm{E}\left|A_{i}\right|^{r} \ell_{s}(\mu, v)
$$

(b) for $s=2$ and $\mu, v \in M_{2}\left(\mu_{0}, c\right)$, we have

$$
\ell_{2}(T \mu, T v) \leq\left(\mathrm{E} \sum_{i=1}^{K} A_{i}^{2}\right)^{1 / 2} \ell_{2}(\mu, v)
$$

(c) for $1 \leq s \leq 2$ and $\mu, v \in M_{s}\left(\mu_{0}, c\right)$, we have

$$
\ell_{s}(T \mu, T \nu) \leq K_{s}\left(\mathrm{E} \sum_{i=1}^{K}\left|A_{i}\right|^{s}\right)^{1 / s} \ell_{s}(\mu, v)
$$

where $K_{1}=1$ and $K_{s}=18 s^{3 / 2}(s-1)^{1 / 2}, 1<s \leq 2$.

For the proof of Lemma 2.1, see [23], [24, Propositions 2 and 3], and, for $s=2$, [26]. In these references the results were stated for the moment class $M_{s}$, but can be extended to the generalized classes $M_{s}\left(\mu_{0}\right)$ and $M_{s}\left(\mu_{0}, c\right)$ considered here. In the cases in which $s=2$ and $0<s<1$, we obtain existence and uniqueness results for additive recursive stochastic equations of the type $T X \stackrel{\mathrm{D}}{=} X$, under the natural contraction condition $\eta_{s}=\mathrm{E} \sum_{i=1}^{K}\left|A_{i}\right|^{s}<1$, in $M_{s}\left(\mu_{0}\right)$ for $0<s \leq 1$ and in $M_{2}\left(\mu_{0}, c\right)$ for $s=2$.

Theorem 2.1. Assume that $\eta_{s}<1$ and consider the stochastic equation

$$
X \stackrel{\mathrm{D}}{=} \sum_{i=1}^{K} A_{i} X_{i}+b
$$

(a) If $0<s \leq 1$ and $\mu_{0} \in M$ satisfies $\ell_{s}\left(\mu_{0}, T \mu_{0}\right)<\infty$, then (2.4) has a unique solution in $M_{s}\left(\mu_{0}\right)$. 
(b) If $s=2, b \in L^{1}, \ell_{2}\left(\mu_{0}, T \mu_{0}\right)<\infty$ for some $\mu_{0} \in M$, and either

$$
\mathrm{E} b=0 \quad \text { and } \quad \mathrm{E} \sum_{i=1}^{K} A_{i}=1
$$

or

$$
\mathrm{E} \sum_{i=1}^{K} A_{i} \neq 1
$$

then either (2.4) has a unique solution in $M_{2}\left(\mu_{0}, c\right)$ for any $c \in \mathbb{R}^{1}$ (under (2.5)), or (2.4) has a unique solution in $M_{2}\left(\mu_{0}, c^{*}\right)$ for $c^{*}:=\mathrm{E} b /\left(1-\mathrm{E} \sum_{i=1}^{K} A_{i}\right)$ (under (2.6)).

Proof. (a) We have to establish that $T$ maps $M_{S}\left(\mu_{0}\right)$ to $M_{s}\left(\mu_{0}\right)$. Let $\mu \in M_{S}\left(\mu_{0}\right)$. Then there exist random variables $X \stackrel{\mathrm{D}}{=} \mu_{0}$ and $Y \stackrel{\mathrm{D}}{=} \mu$ with $\mathrm{E}|X-Y|^{s}<\infty$. Let $\left(X_{i}, Y_{i}\right)$ be i.i.d. random variables with $\left(X_{i}, Y_{i}\right) \stackrel{\mathrm{D}}{=}(X, Y)$. Then

$$
W:=\sum_{i=1}^{K} A_{i} Y_{i}+b \stackrel{\mathrm{D}}{=} T \mu \quad \text { and } \quad V:=\sum_{i=1}^{K} A_{i} X_{i}+b \stackrel{\mathrm{D}}{=} T \mu_{0}
$$

are couplings of $T \mu$ and $T \mu_{0}$ with

$$
\mathrm{E}|W-V|^{s} \leq \sum_{i=1}^{K} \mathrm{E}\left|A_{i}\right|^{s} \mathrm{E}|X-Y|^{s}
$$

(cf. (2.2) for $0<s \leq 1)$. By assumption, $\ell_{s}\left(\mu_{0}, T \mu_{0}\right)<\infty$. Thus, there exist couplings $U$ and $\tilde{U}$ of $\mu_{0}$ and $T \mu_{0}$ with $\mathrm{E}|U-\tilde{U}|^{s}<\infty$. Without loss of generality, we may assume that $\tilde{U}=V$ as defined above (otherwise, we may use a suitable measure-preserving transformation). Thus, $(U, W)$ is a coupling of $\left(\mu_{0}, T \mu\right)$ with $\mathrm{E}|U-W|^{s} \leq \mathrm{E}|U-V|^{s}+\mathrm{E}|V-W|^{s}<\infty$, i.e. $T \mu \in M_{s}\left(\mu_{0}\right)$. The completeness of $\left(M_{s}\left(\mu_{0}\right), \ell_{s}\right)$ is a consequence of the completeness of $L^{s}$. If $\left(\mu_{n}\right) \subset M_{s}\left(\mu_{0}\right)$ is a Cauchy sequence in $M_{s}\left(\mu_{0}\right)$ (and we denote by $F_{n}$ the distribution function of $\mu_{n}$ ), then, by choosing optimal couplings $X_{n}=F_{n}^{-1}(U), n \geq 0$, simultaneously for all $\mu_{n}$, we find that $\left(X_{n}-X_{0}\right)_{n \geq 1}$ is a Cauchy sequence in $L^{s}$ and, thus, has a limit $Z \in L^{s}$. This implies that $\ell_{s}\left(\mu_{n}, \tau\right) \rightarrow 0$, where $\tau \stackrel{\mathrm{D}}{=} X_{0}+Z$. An application of Banach's fixed point theorem using the contraction property in (2.2) now yields the existence and uniqueness of a fixed point in $M_{s}\left(\mu_{0}\right)$.

(b) As in the proof of (a), we have to establish that $T$ maps $M_{2}\left(\mu_{0}, c\right)$ to $M_{2}\left(\mu_{0}, c\right)$. This follows similarly to (a), using conditions (2.5) and (2.6) (as appropriate) to establish that $\int x \mathrm{~d} \mu(x)=c$ implies $\int x \mathrm{~d} T \mu(x)=c$.

Remark 2.1. If $\mu_{0} \in M_{s}$ and $b \in L^{s}, 1 \leq s \leq 2$, then

$$
M_{s}\left(\mu_{0}, c\right) \equiv M_{s}(c):=\left\{\mu \in M_{s}: \int x \mathrm{~d} \mu(x)=c\right\} \subset M_{s},
$$

$M_{s}\left(\mu_{0}\right)=M_{s}$, and the condition $\ell_{s}\left(\mu_{0}, T \mu_{0}\right)<\infty$ is satisfied due to the assumptions on $A_{i}$ and $b$. The contraction and the existence and uniqueness results can respectively be found in this case in [26], for $s=2$, and [24]. The respective extensions to the classes 
$M_{s}\left(\mu_{0}\right)$ and $M_{s}\left(\mu_{0}, c\right) \subset M_{1}$ allow us to consider more general stochastic equations, including, e.g. characterizations of the Cauchy distribution by an equation of the form

$$
A_{1} X_{1}+A_{2} X_{2}+b \stackrel{\mathrm{D}}{=} X
$$

where $b=A_{3} \mathcal{C}(0,1)(\mathcal{C}(0,1)$ being a Cauchy-distributed random variable $), 0 \leq A_{i}, A_{1}+$ $A_{2}+A_{3}=1$, and $\mathrm{E}\left(A_{1}^{s}+A_{2}^{s}\right)<1$ for some $s \leq 1$.

Theorem 2.1(a) then implies that the Cauchy distribution $\mu_{0}=\mathcal{C}(\mu, \sigma)$ is the unique solution to (2.7) in $M_{S}\left(\mu_{0}\right)$.

For $1<s<2$, only the contraction property in (2.3), which has an additional contraction factor $K_{s}>1$, is available. Our next aim is to establish an existence and uniqueness result in $M_{1}$ for this case under the natural contraction condition $\eta_{s}<1$, which extends part (b) of Theorem 2.1.

Theorem 2.2. (Existence and uniqueness in $M_{1}$.) Consider the stochastic equation

$$
X \stackrel{\mathrm{D}}{=} \sum_{i=1}^{K} A_{i} X_{i}+b
$$

(as in (2.4)) and let $1 \leq s \leq 2$. Furthermore, let $\mu_{0} \in M_{1}$ and $b \in L^{1}$ and assume that $\eta_{s}=\mathrm{E} \sum_{i=1}^{K}\left|A_{i}\right|^{s}<1$ and that either (2.5) or (2.6) holds.

If $\ell_{s}\left(\mu_{0}, T \mu_{0}\right)<\infty$ then the stochastic equation (2.4) has a unique solution in $M_{s}\left(\mu_{0}, c\right)$.

Proof. For the proof we establish, in the first step, that the $m$ th iterate, $T^{m}$, of $T$ is, for all $m \geq m_{0}$, a contraction on $M_{s}\left(\mu_{0}, c\right)$, i.e. that $\ell_{s}\left(T^{m} \mu, T^{m} \tau\right) \leq \kappa_{s} \ell_{s}(\mu, \tau)$ for some $\kappa_{s}$, $0<\kappa_{s}<1$, and all $\tau, \mu \in M_{s}\left(\mu_{0}, c\right)$.

We consider the random weighted $K$-ary branching tree, $T_{m}^{X}$, of depth $m$, where each node $\sigma=\sigma_{1} \cdots \sigma_{r}$ (including the root, $\varnothing$ ) is supplied with independent copies, $X_{\sigma}$ and $b_{\sigma}$, of the random variables $X \stackrel{\mathrm{D}}{=} \mu$ and $b$, and the $K$ edges, $e_{1}, \ldots, e_{K}$, leading from $\sigma$ to the successor, $\sigma \sigma_{i}$, of $\sigma$ are supplied with independent copies, $\left(A_{e_{1}}, \ldots, A_{e_{K}}\right)$, of $\left(A_{1}, \ldots, A_{K}\right)$ such that $\left(A_{e_{1}}, \ldots, A_{e_{K}}, b_{\sigma}\right) \stackrel{\mathrm{D}}{=}\left(A_{1}, \ldots, A_{K}, b\right)$ (see, e.g. [29] for this construction). Furthermore, for each node $v=v_{1} \cdots v_{r}$ at level $r$, we define the multiplicative weights $L(v)=A_{v_{1}} \cdots A_{v_{r}}$ along its path $v_{1} \cdots v_{r}$ in the tree, and we define the additively weighted size of the branching tree by

$$
Z_{m}:=\sum_{|\sigma|=m} L(\sigma) X_{\sigma}+\sum_{i=1}^{m-1} \sum_{|\nu|=i} L(\nu) b_{\nu}, \quad m \in \mathbb{N} .
$$

Let $\tau, \mu \in M_{s}\left(\mu_{0}, c\right)$, let $X \stackrel{\mathrm{D}}{=} \mu$ and $Y \stackrel{\mathrm{D}}{=} \tau$ with $\mathrm{E} X=\mathrm{E} Y$ and $\mathrm{E}|X-Y|^{s}<\infty$, and let $T_{m}^{Y}$ be the induced random weighted branching tree with i.i.d. copies, $Y_{\sigma}$, of $Y$ at the nodes and with the same random weights $\left(A_{\sigma}, b_{\sigma}\right)$ on the edges as in the tree $T_{m}^{X}$. The random variables $\left\{\left(X_{\sigma}, Y_{\sigma}\right)\right\}$ are i.i.d. and such that $\left(X_{\sigma}, Y_{\sigma}\right) \stackrel{\mathrm{D}}{=}(X, Y)$. Denote the corresponding additively weighted size by

$$
W_{m}:=\sum_{|\sigma|=m} L(\sigma) Y_{\sigma}+\sum_{i=1}^{m-1} \sum_{|\nu|=i} L(v) b_{v}, \quad m \in \mathbb{N} .
$$


Then $Z_{m}$ and $W_{m}$ respectively display the recursive structures

$$
Z_{m} \stackrel{\mathrm{D}}{=} \sum_{i=1}^{K} A_{i} Z_{m-1}^{(i)}+b, \quad W_{m} \stackrel{\mathrm{D}}{=} \sum_{i=1}^{K} A_{i} W_{m-1}^{(i)}+b,
$$

where $\left(Z_{m-1}^{(i)}\right)$ and $\left(W_{m-1}^{(i)}\right)$ are respectively i.i.d. copies of $Z_{m-1}$ and $W_{m-1}$. This recursive structure is obtained by splitting the tree at the root. The weighted sizes $Z_{m}$ and $W_{m}$ are versions of the $m$ th iterate of the distributional operator $T$ :

$$
Z_{m} \stackrel{\mathrm{D}}{=} T^{m} X, \quad W_{m} \stackrel{\mathrm{D}}{=} T^{m} Y .
$$

By the multiplicative structure presented, and using the independence assumptions, from the Woyczynski inequality (see (2.3)) we obtain

$$
\begin{aligned}
L_{s}^{s}\left(Z_{m}, W_{m}\right) & =\mathrm{E}\left|\sum_{|\sigma|=m} L(\sigma)\left(X_{\sigma}-Y_{\sigma}\right)\right|^{s} \\
& \leq K_{s} \mathrm{E} \sum_{|\sigma|=m}|L(\sigma)|^{s} \mathrm{E}|X-Y|^{s} \\
& =K_{s}\left(\mathrm{E} \sum_{i=1}^{K}\left|A_{i}\right|^{s}\right)^{m} \mathrm{E}|X-Y|^{s} \\
& =K_{s} \eta_{s}^{m} \mathrm{E}|X-Y|^{s} .
\end{aligned}
$$

For this estimate, the equality of first moments is needed. Passing to the minimal $L_{s}$-metric $\ell_{s}$, we obtain

$$
\ell_{s}\left(T^{m} X, T^{m} Y\right) \leq K_{s} \eta_{s}^{m} \ell_{s}(\mu, \tau) .
$$

For $m \geq m_{0}, K_{s} \eta_{s}^{m} \leq K_{s} \eta_{s}^{m_{0}}=: \kappa_{s}<1$, i.e. the iterated operator $T^{m}$ is a contraction with respect to $\ell_{s}$ on $M_{s}\left(\mu_{0}, c\right)$.

By assumption, $\ell_{s}\left(\mu_{0}, T \mu_{0}\right)<\infty$ and, thus, as in the proof of part (b) of Theorem 2.1, for $s=2$ we find that $T$ maps $M_{s}\left(\mu_{0}, c\right)$ to $M_{s}\left(\mu_{0}, c\right)$. By the triangle inequality, this implies that $\ell_{s}\left(\mu_{0}, T^{m} \mu_{0}\right)<\infty$. Thus, $\mu_{0}, T^{m} \mu_{0}, T^{2 m} \mu_{0}, \ldots$ is a Cauchy sequence in $M_{s}\left(\mu_{0}, c\right)$ and, so, converges to some limit $\mu^{*} \in M_{s}\left(\mu_{0}, c\right): \ell_{s}\left(T^{k m} \mu_{0}, \mu^{*}\right) \rightarrow 0$. For any $r, 1 \leq r \leq m$, we obtain

$$
\ell_{s}\left(T^{k m} \mu_{0}, T^{k m+r} \mu_{0}\right) \leq \kappa_{s}^{k} \ell_{s}\left(\mu_{0}, T^{r} \mu_{0}\right) \rightarrow 0 ;
$$

thus, the triangle inequality implies that $\ell_{s}\left(\mu^{*}, T^{r} \mu^{*}\right)=0,1 \leq r \leq m$, and $T^{n} \mu_{0}$ converges to $\mu^{*}$ and $\mu^{*}$ is a fixed point of $T$ in $M_{s}\left(\mu_{0}, c\right)$.

Uniqueness of the fixed point follows from the estimate in (2.12) if applied to two solutions $X$ and $Y$ of (2.4).

Remark 2.2. As for $s=2$, the additional assumption that $b \in L^{s}$ implies the condition $\ell_{s}\left(\mu_{0}, T \mu_{0}\right)<\infty$ if $\mu_{0} \in M_{s}$.

We can state a corresponding existence and uniqueness result with respect to the Zolotarev metric $\zeta_{s}$ for any $s>0$. Here the proof is much simpler and does not require consideration of the iterated operators or the weighted branching processes. The metric $\zeta_{s}(\mu, \nu)$ is defined, for $s=m+\alpha, m \in \mathbb{N}_{0}, 0<\alpha \leq 1$, and $X \stackrel{\mathrm{D}}{=} \mu$ and $Y \stackrel{\mathrm{D}}{=} v$, by

$$
\zeta_{s}(\mu, v)=\sup \left\{\mathrm{E}(f(X)-f(Y)): f \in \mathcal{F}_{s}\right\},
$$


where

$$
\mathcal{F}_{s}=\left\{f \in \mathcal{C}^{m}(\mathbb{R}, \mathbb{R}):\left\|f^{(m)}(x)-f^{(m)}(y)\right\| \leq|x-y|^{\alpha}\right\} .
$$

Finiteness of $\zeta_{s}(\mu, v)$ implies equality of the first $m$ difference moments: $\mathrm{E}\left(X^{r}-Y^{r}\right)=0,1 \leq$ $r \leq m$.

Proposition 2.1. Let $s>0$ and let $\mu_{0} \in M$ be a probability measure such that $\eta_{s}=$ $\mathrm{E} \sum_{i=1}^{K}\left|A_{i}\right|^{s}<1$ and $\zeta_{s}\left(\mu_{0}, T \mu_{0}\right)<\infty$. Then the additive stochastic equation (2.4) has a unique solution in $M_{s}^{\zeta}\left(\mu_{0}\right)=\left\{\mu \in M: \zeta_{s}\left(\mu, \mu_{0}\right)<\infty\right\}$.

Proof. For $\mu, v \in M_{s}^{\zeta}\left(\mu_{0}\right)$, we have

$$
\zeta_{s}(T \mu, T \nu) \leq\left(\sum_{i=1}^{K} \mathrm{E}\left|A_{i}\right|^{s}\right) \zeta_{s}(\mu, v)
$$

(see, e.g. [24, Proposition 1]). The assumption $\zeta_{s}\left(\mu_{0}, T \mu_{0}\right)<\infty$ implies, by the triangle inequality, that $T$ maps $M_{s}^{\zeta}\left(\mu_{0}\right)$ to $M_{s}^{\zeta}\left(\mu_{0}\right)$. Thus, $\left\{T^{n} \mu_{0}\right\}_{n \in \mathbb{N}}$ is a Cauchy sequence in $M_{s}^{\zeta}\left(\mu_{0}\right)$, which implies the existence of a fixed point by completeness of $\left(M_{s}^{\zeta}\left(\mu_{0}\right), \zeta_{s}\right)$. The uniqueness part is as in Theorem 2.2.

Remark 2.3. In general, the finiteness condition, $\zeta_{s}\left(\mu_{0}, T \mu_{0}\right)<\infty$, of Proposition 2.1 for the Zolotarev metric $\zeta_{s}$ is not easy to check. For $s \in \mathbb{N}$ there are upper bounds of $\zeta_{s}$ in terms of the difference pseudomoments

$$
\kappa_{s}(X, Y)=s \int|x|^{s-1}\left|F_{X}(x)-F_{Y}(x)\right| \mathrm{d} x
$$

(where $F_{X}$ and $F_{Y}$ are the respective distribution functions of $X$ and $Y$ ) if the first $s-1$ moments coincide, but for $s \notin \mathbb{N}$ only estimates for the $\zeta_{s}$-metric including absolute pseudomoments are available. There have been developed several alternative probability metrics, $\mu_{s}$, which allow estimates as in (2.13) and simultaneously admit upper bounds in terms of difference pseudomoments (see, e.g. [22]). However, in comparison, the estimate of $\ell_{s}\left(\mu_{0}, T \mu_{0}\right)$ with respect to the $\ell_{s}$-metric is particularly simple and will be very useful in the following part of the paper.

As a consequence of Proposition 2.1, we find that, given the contraction condition $\eta_{s}<1$, demonstrating the existence of a fixed point is equivalent to finding an element $\mu_{0} \in M$ such that $\zeta_{s}\left(\mu_{0}, T \mu_{0}\right)<\infty$. With respect to the $\ell_{s}$-metrics, for $0<s \leq 1$ the same is true in $M_{s}\left(\mu_{0}\right)$. For the interesting case, $1 \leq s \leq 2$, we obtain a characterization of fixed points in $M_{s}\left(\mu_{0}, c\right) \subset M_{1}$. In the next step we want to remove the first moment condition for the $\ell_{s}$-metrics. To that end we introduce, for any $\mu_{0} \in M$,

$$
\begin{aligned}
& M_{s}^{0}\left(\mu_{0}\right)=\left\{\mu \in M: \text { there exist random variables } X \stackrel{\mathrm{D}}{=} \mu \text { and } Y \stackrel{\mathrm{D}}{=} \mu_{0}\right. \\
& \text { such that } \left.\mathrm{E}(X-Y)=0 \text { and } \mathrm{E}|X-Y|^{s}<\infty\right\} .
\end{aligned}
$$

On $M_{s}^{0}\left(\mu_{0}\right)$ we define the modified $\ell_{s}$-metric

$$
\ell_{s}^{0}(\mu, v)=\inf \left\{\left(\mathrm{E}|X-Y|^{s}\right)^{1 / s \wedge 1}: X \stackrel{\mathrm{D}}{=} \mu, Y \stackrel{\mathrm{D}}{=} v, \mathrm{E}(X-Y)=0, \mathrm{E}|X-Y|^{s}<\infty\right\} .
$$

Since $M_{s}^{0}\left(\mu_{0}\right) \subset M_{s}\left(\mu_{0}\right)$, we obtain $\ell_{s}(\mu, v) \leq \ell_{s}^{0}(\mu, v)$ and $\ell_{s}^{0}$ satisfies the triangle inequality. Finiteness of $\ell_{s}^{0}(\mu, v)$ needs a more stringent coupling than does finiteness of $\ell_{s}(\mu, v)$. In the 
next theorem we will see that this finiteness is sufficient for a general existence result for solutions to the stochastic recursive equation $X \stackrel{\mathrm{D}}{=} \sum_{i=1}^{K} A_{i} X_{i}+b$ using $\ell_{s}$-type estimates.

Theorem 2.3. (Existence and uniqueness in M.) Let $1 \leq s \leq 2$, let $\mu_{0} \in M$ satisfy $\ell_{s}^{0}\left(\mu_{0}, T \mu_{0}\right)<\infty$, and assume that $\eta_{s}=\mathrm{E} \sum_{i=1}^{K}\left|A_{i}\right|^{s}<1$. Then the stochastic equation (2.4) has a unique solution in $M_{s}^{0}\left(\mu_{0}\right)$.

Proof. We first establish that $T$ maps $M_{s}^{0}\left(\mu_{0}\right)$ to $M_{s}^{0}\left(\mu_{0}\right)$. Let $\mu \in M_{s}^{0}\left(\mu_{0}\right)$. For random variables $X$ and $Y$, by $X \approx Y$ we indicate that $\mathrm{E}(X-Y)=0$ and $\mathrm{E}|X-Y|^{s}<\infty$. By assumption, there exist random variables $X \stackrel{\mathrm{D}}{=} \mu_{0}$ and $Y \stackrel{\mathrm{D}}{=} T \mu_{0}$ such that $X \approx Y$. Let $\left(X_{i}, Y_{i}\right)$ be i.i.d. copies of $(X, Y)$. Furthermore, define couplings of $T \mu$ and $T \mu_{0}$ by

$$
Z:=\sum_{i=1}^{K} A_{i} Y_{i}+b \stackrel{\mathrm{D}}{=} T \mu, \quad W:=\sum_{i=1}^{K} A_{i} X_{i}+b \stackrel{\mathrm{D}}{=} T \mu_{0} .
$$

Then

$$
\begin{gathered}
\mathrm{E}(Z-W)=\sum_{i=1}^{K} \mathrm{E} A_{i} \mathrm{E}(Y-X)=0, \\
\mathrm{E}|Z-W|^{s} \leq K_{s}^{s} \eta_{s} \mathrm{E}|Y-X|^{s}<\infty,
\end{gathered}
$$

i.e. $Z \approx W$. Since $\ell_{s}^{0}\left(\mu_{0}, T \mu_{0}\right)<\infty$, for $\ell_{s}^{0}$ we obtain

$$
\ell_{s}^{0}\left(\mu_{0}, T \mu\right) \leq \ell_{s}^{0}\left(\mu_{0}, T \mu_{0}\right)+\ell_{s}^{0}\left(T \mu_{0}, T \mu\right) \leq \mathrm{E}|X-Y|^{s}+\mathrm{E}|Z-W|^{s}<\infty,
$$

i.e. $T \mu \in M_{s}^{0}\left(\mu_{0}\right)$, by the triangle inequality. Now we can follow the proof of Theorem 2.2 using the branching tree construction with $Z_{k m} \stackrel{\mathrm{D}}{=} T^{k m} \mu_{0}$ and $W_{k m+r} \stackrel{\mathrm{D}}{=} T^{k m+r} \mu_{0}$ (cf. (2.8), (2.9), and (2.10)) and with i.i.d. couplings, $\left(Y_{\sigma}, X_{\sigma}\right)$, of $\left(\mu_{0}, T^{r} \mu_{0}\right)$ such that $Y_{\sigma} \approx X_{\sigma}$. Then, as in (2.11), for any $r \geq 1$ we find that

$$
L_{s}^{s}\left(Z_{k m}, W_{k m+r}\right) \leq K_{s}^{s}\left(\eta_{s}^{m}\right)^{k} \mathrm{E}|Y-X|^{s} \rightarrow 0 \quad \text { as } k \rightarrow \infty,
$$

where $(X, Y) \stackrel{\mathrm{D}}{=}\left(X_{\sigma}, Y_{\sigma}\right) \stackrel{\mathrm{D}}{=}\left(T^{r} \mu_{0}, \mu_{0}\right)$. In particular, $\mu_{0}, T^{m} \mu_{0}, T^{2 m} \mu_{0}, \ldots$ is a Cauchy sequence in $M_{s}^{0}\left(\mu_{0}\right)$ with corresponding couplings $X_{0}, X_{1}=Z_{m}, X_{2}=Z_{2 m}, \ldots$.

The related differences, $X_{k}-X_{0}$, are a Cauchy sequence in $L^{s}(0)$ (i.e. they have mean 0 ) and, thus, converge to some limit $Z \in L^{s}(0)$ (we write this as $X_{k}-X_{0} \rightarrow_{L^{s}} Z$ ). This implies that $X_{k} \rightarrow L^{s} Z+X_{0}$. Thus, with $\mu^{*} \stackrel{\mathrm{D}}{=} Z+X_{0}$, we obtain

$$
\ell_{s}^{0}\left(T^{k m} \mu_{0}, \mu^{*}\right) \leq \mathrm{E}\left|X_{k}-\left(Z+X_{0}\right)\right|^{s} \rightarrow 0 .
$$

This argument yields the completeness of $\left(M_{s}^{0}\left(\mu_{0}\right), \ell_{s}^{0}\right)$. From (2.14) we conclude that

$$
\ell_{s}^{0}\left(\mu^{*}, T^{r} \mu^{*}\right)=0, \quad 1 \leq r \leq m,
$$

and, thus, $\mu^{*}$ is a fixed point of $T$ in $M_{s}^{0}\left(\mu_{0}\right)$. Uniqueness follows from application of the estimate (2.14) to two solutions, $\mu$ and $\nu$, to the stochastic equation, and use of the corresponding weighted branching tree construction with sizes $Z_{n}$ and $W_{n}$ and couplings $X \stackrel{\mathrm{D}}{=} \mu$ and $Y \stackrel{\mathrm{D}}{=} v$ such that $X \approx Y$.

As a consequence of Theorems 2.3 and 2.1, we obtain the following characterization of the existence of solutions in the class, $M$, of all distributions. The necessity part of the following characterization is obvious. 
Corollary 2.1. Let $0<s \leq 2$ and $\eta_{s}=\mathrm{E} \sum_{i=1}^{K}\left|A_{i}\right|^{s}<1$. Then the stochastic equation

$$
X \stackrel{\mathrm{D}}{=} \sum_{i=1}^{K} A_{i} X_{i}+b
$$

has a solution in $M$ if and only if there exists some $\mu_{0} \in M$ such that

$$
\begin{array}{ll}
\ell_{s}\left(\mu_{0}, T \mu_{0}\right)<\infty & \text { if } 0<s \leq 1, \\
\ell_{s}^{0}\left(\mu_{0}, T \mu_{0}\right)<\infty & \text { if } 1<s \leq 2 .
\end{array}
$$

Remark 2.4. In particular, the extended contraction results for the $\ell_{s}$-metrics allow us to characterize stable distributions as unique solutions to the associated stochastic equations in $M_{s}\left(\mu_{0}\right)$ or $M_{s}^{0}\left(\mu_{0}\right)$. Let $0<\alpha<2$, let $U$ be uniformly distributed on [0,1], and let $X^{*} \stackrel{\mathrm{D}}{=} \mu_{0}=\delta(\alpha)$ be a symmetric, stable distribution with index $\alpha$ and scale factor $c$, with characteristic function

$$
\ln \varphi_{\&(\alpha)}(t)=-c|t|^{\alpha} .
$$

Then $X^{*}$ is the unique solution to the stochastic recursion

$$
X \stackrel{\mathrm{D}}{=} U^{1 / \alpha} X_{1}+(1-U)^{1 / \alpha} X_{2}
$$

in $M_{s}\left(\mu_{0}\right)$, if $0<\alpha<s \leq 1$, or in $M_{s}^{0}\left(\mu_{0}\right)$, if $1 \leq \alpha<s \leq 2$.

\section{Homogeneous and inhomogeneous additive recursive equations}

In this section we obtain, as an application of the contraction results established in Section 2, a one-to-one relationship between solutions to homogeneous and, respectively, inhomogeneous linear stochastic equations. Consider the inhomogeneous equation

$$
X \stackrel{\mathrm{D}}{=} \sum_{i=1}^{K} A_{i} X_{i}+b,
$$

with induced operator $T$ on $M$, and the corresponding homogeneous equation

$$
X \stackrel{\mathrm{D}}{=} \sum_{i=1}^{K} A_{i} X_{i}
$$

with induced operator $T_{0}$. To establish a one-to-one relationship we assume that $b \in L^{s}$ and that the natural contraction condition, $\eta_{s}=\mathrm{E} \sum_{i=1}^{K}\left|A_{i}\right|^{s}<1$, holds. In Section 2 we obtained various conditions on the existence and uniqueness of solutions to (3.1) and (3.2).

Theorem 3.1. (Homogeneous and inhomogeneous equations.) Let $0<s \leq 2$ and $A_{i}, b \in L^{s}$ such that $\eta_{s}=\mathrm{E} \sum_{i=1}^{K}\left|A_{i}\right|^{s}<1$ and $\mathrm{E} b=0$ for $1<s \leq 2$. Then the following equivalence holds.

(a) For any solution $\mu_{0}$ to the homogeneous equation $T_{0} \mu \stackrel{\mathrm{D}}{=} \mu_{0}$, there exists exactly one solution, $\mu^{*}$, to the inhomogeneous equation $T \mu \stackrel{\mathrm{D}}{=} \mu$, such that

$$
\mu^{*} \in \begin{cases}M_{s}\left(\mu_{0}\right) & \text { if } 0<s \leq 1 \\ M_{s}^{0}\left(\mu_{0}\right) & \text { if } 1<s \leq 2\end{cases}
$$


(b) For any solution $\mu^{*}$ to the inhomogeneous equation $T \mu \stackrel{\mathrm{D}}{=} \mu$, there exists exactly one solution, $\mu_{0}$, to the homogeneous equation $T_{0} \mu \stackrel{\mathrm{D}}{=} \mu$, such that

$$
\mu_{0} \in \begin{cases}M_{s}\left(\mu^{*}\right) & \text { if } 0<s \leq 1 \\ M_{s}^{0}\left(\mu_{0}\right) & \text { if } 1<s \leq 2\end{cases}
$$

Proof. (a) If $\mu_{0}$ is a solution to the homogeneous equation $T_{0} \mu_{0} \stackrel{\mathrm{D}}{=} \mu_{0}$, then we obtain a coupling of $\mu_{0}$ and $T \mu_{0}$ from $Y_{0}:=\sum_{i=1}^{K} A_{i} Y_{i}$, where $\left(Y_{i}\right)$ are i.i.d., $Y_{i} \stackrel{\mathrm{D}}{=} \mu_{0}$, and $X^{*}:=$ $\sum_{i=1}^{K} A_{i} Y_{i}+b=Y_{0}+b$. This implies that $\mathrm{E}\left|X^{*}-Y_{0}\right|^{s}=\mathrm{E}|b|^{s}<\infty$ and, thus, that $\ell_{s}\left(\mu_{0}, T \mu_{0}\right)<\infty$ for $0<s \leq 1$ and $\ell_{s}^{0}\left(\mu_{0}, T \mu_{0}\right)<\infty$ for $1<s \leq 2$, using the additional assumption that $\mathrm{E} b=0$ in the latter case. From Theorem 2.1(a) we obtain a unique solution, $\mu^{*} \in M_{s}\left(\mu_{0}\right)$, to $T \mu \stackrel{\mathrm{D}}{=} \mu$ for $0<s \leq 1$, while Theorem 2.3 implies the existence of a unique solution, $\mu^{*} \in M_{s}^{0}\left(\mu_{0}\right)$, to $T \mu \stackrel{\mathrm{D}}{=} \mu$ for $1<s \leq 2$.

(b) The converse statement is proved similarly. If $\mu^{*}$ is a solution to the inhomogeneous equation $T \mu \stackrel{\mathrm{D}}{=} \mu$, then let $\left(X_{i}\right)$ be i.i.d. random variables with $X_{i} \stackrel{\mathrm{D}}{=} \mu^{*}$. Then $X^{*}:=\sum_{i=1}^{K} A_{i} X_{i}+b$ and $Y_{0}:=\sum_{i=1}^{K} A_{i} X_{i}$ define a coupling of $\mu^{*}$ and $T_{0} \mu^{*}$ such that $\mathrm{E}\left|Y_{0}-X^{*}\right|^{s}=\mathrm{E}|b|^{s}<\infty$, $\ell_{s}\left(\mu^{*}, T_{0} \mu^{*}\right)<\infty$ for $0<s \leq 1$, and $\ell_{s}^{0}\left(\mu^{*}, T_{0} \mu^{*}\right) \leq \mathrm{E}\left|Y_{0}-X^{*}\right|^{s}<\infty$ for $1<s \leq 2$. Furthermore, $T_{0} \mu^{*} \in M_{s}^{0}\left(\mu^{*}\right)$. Thus, again by Theorems 2.1(a) and 2.3, we obtain a unique solution, $\mu_{0}$, to the homogeneous equation $T_{0} \mu=\mu$, with

$$
\begin{array}{ll}
\mu_{0} \in M_{s}\left(\mu^{*}\right) & \text { if } 0<s \leq 1, \\
\mu_{0} \in M_{s}^{0}\left(\mu^{*}\right) & \text { if } 1<s \leq 2 .
\end{array}
$$

Remark 3.1. (a) Let $\mathcal{L}_{0}$ and $\mathcal{L}$ respectively denote the solution sets of the homogeneous and inhomogeneous equations (either in terms of distributions or in terms of random variables). If $Y \in \mathcal{L}_{0} \cap L^{1}, \mathrm{E} Y=c$, and $1 \leq s \leq 2$, then with $\mu_{0} \stackrel{\mathrm{D}}{=} Y$ we have $M_{s}^{0}\left(\mu_{0}\right)=M_{s}\left(\mu_{0}, c\right)$. We obtain, as a consequence of Theorems 2.3 and 3.1, the existence and uniqueness of solutions in $M_{s}\left(\mu_{0}, c\right)$, as in Theorem 2.2, where we had to specify the first moments of the solutions. The remarkable feature of Theorem 3.1 is that it establishes a one-to-one relationship between $\mathcal{L}_{0}$ and $\mathcal{L}$ without any assumptions on the moments of the solutions, $\mu_{0}$ and $\mu^{*}$, in $\mathcal{L}_{0}$ and, respectively, $\mathcal{L}$. The existence result based on the Zolotarev metric (Proposition 2.1) does not allow us to draw the conclusion Theorem 3.1 does since the finiteness condition, $\zeta_{s}\left(\mu_{0}, T \mu_{0}\right)<\infty$, for a fixed point $\mu_{0}$ of the homogeneous equation in general needs further moment assumptions on $\mu_{0}$.

(b) Fill and Janson [9] characterized the set of all solutions to the Quicksort recursion

$$
X \stackrel{\mathrm{D}}{=} U X_{1}+(1-U) X_{2}+C(U),
$$

with $C(U)=2 U \log U+2(1-U) \log (1-U)+1$, by

$$
\mathcal{L}=X^{*} \oplus \mathcal{C},
$$

where $X^{*}$ is the unique solution to the inhomogeneous equation - the Quicksort distribution with finite 2 nd moment; $\mathcal{C}=\left\{C\left(\mu, \sigma^{2}\right): \mu \in \mathbb{R}^{1}, \sigma^{2} \geq 0\right\}$ is the set of Cauchy distributions $C\left(\mu, \sigma^{2}\right)$ with location parameter $\mu$ and scale parameter $\sigma$, such that $C(\mu, 0)=\varepsilon_{\mu}$; and ' $\oplus$ ' denotes independent sums. Their method of proof, when applied to the homogeneous equation, yields

$$
\mathcal{L}_{0}=\mathcal{C},
$$


i.e. $\mathcal{C}$ is the set of all solutions to the homogeneous equation. In this case the relation between the homogeneous solutions and the inhomogeneous solutions is explicit and simple. According to the explicit relationship, knowledge of the solution set in the homogeneous case in (3.4) directly yields, by the equivalence result of Theorem 3.1, the equality in (3.3) in the inhomogeneous case.

Corollary 3.1. (Quicksort-type equation.) Let $U$ be $\mathrm{U}[0,1]$-distributed and let $b \in L^{s}$ under condition (2.5) or condition (2.6) if $1 \leq s \leq 2$. Then the Quicksort-type equation

$$
X \stackrel{\mathrm{D}}{=} U X_{1}+(1-U) X_{2}+b
$$

has a unique solution, $\tilde{X}$, in $M_{S}(c)$ if (2.5) applies, or in $M_{S}\left(c^{*}\right)$ if (2.6) applies, and the set of all solutions is given by $\mathcal{L}=\tilde{X} \oplus \mathcal{C}$.

Proof. Using (3.4), the proof follows from Theorems 2.1, 2.2, and 3.1.

\section{Max-recursive sequences}

In this section we consider max-recursive equations of the kind

$$
X \stackrel{\mathrm{D}}{=} \bigvee_{r=1}^{K}\left(A_{r} X_{r}+b_{r}\right),
$$

where $\left(X_{i}\right)$ are i.i.d. copies of $X$ and $A_{i}$ and $b_{i}$ are random coefficients independent of $\left(X_{r}\right)$. The right-hand side of (4.1) induces an operator $T: M \rightarrow M$ defined, for $Q \in M$ and $X \stackrel{\mathrm{D}}{=} Q$, by

$$
T Q=T X \stackrel{\mathrm{D}}{=} \mathcal{L}\left(\bigvee_{r=1}^{K}\left(A_{r} X_{r}+b_{r}\right)\right) .
$$

If $A_{r}, b_{r} \in L^{s}$ and $\mathcal{L}(X) \in M_{s}$, then $T X \in M_{s}$ and, thus, $T$ can be considered as an operator from $M_{s}$ to $M_{s}$. The following existence and uniqueness result for max-recursive equations was stated in [21], based on the contraction property in (1.5). Note that in the max case any $s>0$ is allowed.

Theorem 4.1. (Existence and uniqueness for max-recursive equations [21].) For some $s>0$, let $A_{i}, b_{i} \in L^{s}$ and let $\mu_{0} \in M$ be such that $\ell_{s}\left(\mu_{0}, T \mu_{0}\right)<\infty$ and $\eta_{s}:=\mathrm{E} \sum_{r=1}^{K}\left|A_{r}\right|^{s}<1$. Then the stochastic max-recursive equation $X \stackrel{\mathrm{D}}{=} \bigvee_{r=1}^{K}\left(A_{r} X_{r}+b_{r}\right)$ has a unique solution in $M_{s}\left(\mu_{0}\right)$.

Similarly to the sum case, we obtain as a consequence a one-to-one relation between the set, $\mathcal{L}$, of solutions to the inhomogeneous equation

$$
X \stackrel{\mathrm{D}}{=} \bigvee_{r=1}^{K}\left(A_{r} X_{r}+b_{r}\right)
$$

and the set, $\mathcal{L}_{0}$, of solutions to the homogeneous equation

$$
Y \stackrel{\mathrm{D}}{=} \bigvee_{r=1}^{K} A_{r} X_{r} .
$$

We denote the corresponding distributional operators by $T$ and $T_{0}$. 
Theorem 4.2. (Equivalence theorem.) Let $A_{r}, b_{r} \in L^{s}$ for some $s>0$ and assume that $\eta_{s}=\mathrm{E} \sum_{r=1}^{K}\left|A_{r}\right|^{s}<1$. The following statements then hold.

(a) The inhomogeneous max-recursive equation (4.2) has a unique solution in $M_{s}$.

(b) For any solution $Y_{0} \in \mathcal{L}_{0}$ to the homogeneous max-recursive equation (4.3), there exists exactly one solution, $X^{*}$, to the inhomogeneous max-recursive equation (4.2) such that $\ell_{s}\left(X^{*}, Y_{0}\right)<\infty$, i.e. $\mathcal{L}\left(X^{*}\right) \in M_{s}\left(\mu_{0}\right)$ with $\mu_{0}=\mathcal{L}\left(Y_{0}\right)$.

(c) For any solution $X^{*}$ to the inhomogeneous equation (4.2), there exists exactly one solution, $Y_{0}$, to the homogeneous equation (4.3) with $\ell_{s}\left(X^{*}, Y_{0}\right)<\infty$.

Proof. (a) The proof follows from Theorem 4.1. Let $\mu_{0}=\varepsilon_{0}$. Then

$$
\ell_{s}\left(\mu_{0}, T \mu_{0}\right) \leq \mathrm{E} \max _{r}\left|b_{r}\right|^{s} \leq \sum_{r=1}^{K} \mathrm{E}\left|b_{r}\right|^{s}<\infty .
$$

Furthermore, $M_{s}\left(\mu_{0}\right)=M_{s}$ and, thus, (a) follows from Theorem 4.1.

(b) If $Y_{0} \in \mathcal{L}_{0}$ and $\mu_{0}=\mathcal{L}\left(Y_{0}\right)$ then let $\left(X_{i}\right)$ be independent random variables with $X_{i} \stackrel{\mathrm{D}}{=} \mu_{0}$ and consider the coupling $X:=\bigvee_{r=1}^{K} A_{r} X_{r}$ and $W:=\bigvee_{r=1}^{K}\left(A_{r} X_{r}+b_{r}\right)$ of $\mu_{0}$ and $T \mu_{0}$. From Lemma 3.1 of [21], we then obtain

$$
\ell_{s}\left(\mu_{0}, T \mu_{0}\right) \leq\left(\mathrm{E}|X-W|^{s}\right)^{1 / s \wedge 1} \leq\left(\mathrm{E} \sum_{r=1}^{K}\left|b_{r}\right|^{s}\right)^{1 / s \wedge 1}<\infty .
$$

Theorem 4.1 implies the existence and uniqueness of a solution, $\mu^{*}$, to the inhomogeneous equation (4.2) in $M_{S}\left(\mu_{0}\right)$.

(c) Conversely, let $X^{*}$ be a solution to (4.2) with $X^{*} \stackrel{\mathrm{D}}{=} \mu^{*}$. Furthermore, let $\left(X_{i}\right)$ be i.i.d. with $X_{i} \stackrel{\mathrm{D}}{=} \mu^{*}$ and consider the coupling $X:=\bigvee_{r=1}^{K} A_{r} X_{r}$ and $W:=\bigvee_{r=1}^{K}\left(A_{r} X_{r}+b_{r}\right)$ of $T_{0} \mu^{*}$ and $\mu^{*}$ (as $\left.W \stackrel{\mathrm{D}}{=} \mu^{*}\right)$. Then

$$
\ell_{s}\left(\mu^{*}, T_{0} \mu^{*}\right) \leq\left(\mathrm{E}|X-W|^{s}\right)^{1 / s \wedge 1} \leq\left(\mathrm{E} \sum_{r=1}^{K}\left|b_{r}\right|^{s}\right)^{1 / s \wedge 1}<\infty
$$

and Theorem 4.1 implies the result.

According to Theorem 4.2, there is a one-to-one relationship between the set, $\mathcal{L}$, of solutions to the inhomogeneous max-recursive equation and the set, $\mathscr{L}_{0}$, of solutions to the homogeneous max-recursive equation. In the case of nonnegative coefficients $A_{j} \geq 0$, the homogeneous maxrecursive equation

$$
X \stackrel{\mathrm{D}}{=} \bigvee_{j=1}^{K} A_{j} X_{j}
$$

can be related to the homogeneous additive recursive equation

$$
W^{(\alpha)} \stackrel{\mathrm{D}}{=} \sum A_{j}^{\alpha} W_{j}^{(\alpha)},
$$

where $\alpha$ is chosen such that $\mathrm{E} \sum_{j=1}^{K} A_{j}^{\alpha}=1$. This equation has been studied in detail in the literature. Equation (4.5) has a solution if and only if $\mathrm{E} \sum_{j=1}^{K} A_{j}^{\alpha} \ln A_{j} \leq 0$ (see [3], [4], and [17]). 
Let

$$
K F(t)=\mathrm{E} \prod_{j} F\left(\frac{t}{A_{j}}\right)
$$

denote the operator on the distribution functions $F$ corresponding to (4.4). Rösler [28] showed that, for any nonnegative solution $W^{(\alpha)}$ to the additive equation (4.5),

$$
F_{0}(t):=\mathrm{E} \exp \left(-W^{(\alpha)} t^{-\alpha}\right)
$$

is a distribution function and $K F_{0}=F_{0}$, i.e. $F_{0}$ is a solution to the max-recursive equation (4.5) (see also [16]). We can interpret Rösler's analytic construction stochastically as an analogue of a transformation of Guivarc'h [11] given (in the latter paper) for the sum case.

Proposition 4.1. (Additive and max-recursive equations.) Let $A_{j} \geq 0$, let $\mathrm{E} \sum_{j=1}^{K} A_{j}^{\alpha}=1$, and let $W^{(\alpha)}$ be a nonnegative solution to the additive stochastic equation (4.5). Furthermore, let $Z^{(\alpha)}$ be Weibull distributed with parameter $\alpha$, i.e. $F_{Z^{(\alpha)}}(x)=\exp \left(-1 / x^{\alpha}\right), x>0$. Then the random scaled Weibull variable $X:=\left(W^{(\alpha)}\right)^{1 / \alpha} Z^{(\alpha)}$ is a solution to the max-recursive equation (4.4).

Proof. We verify that the distribution function of $X$ is identical to Rösler's [28] distribution function $F_{0}$ in (4.6):

$$
\begin{aligned}
F_{X}(t) & =\mathrm{P}(X \leq t) \\
& =\mathrm{P}\left(\left(W^{(\alpha)}\right)^{1 / \alpha} Z^{(\alpha)} \leq t\right) \\
& =\mathrm{E} F_{Z^{(\alpha)}}\left(\frac{t}{\left(W^{(\alpha)}\right)^{1 / \alpha}}\right) \\
& =\mathrm{E} \exp \left(-W^{(\alpha)} / t^{\alpha}\right) \\
& =F_{0}(t) .
\end{aligned}
$$

Alternatively, we may use the max-stability of the Weibull distribution. Let $X_{j}=\left(W_{j}^{(\alpha)}\right)^{1 / \alpha} Z_{j}^{(\alpha)}$ be i.i.d. copies of $X$. Then

$$
\begin{aligned}
\bigvee_{j=1}^{K} A_{j} X_{j} & =\bigvee_{j=1}^{K} A_{j}\left(W_{j}^{(\alpha)}\right)^{1 / \alpha} Z_{j}^{(\alpha)} \\
& \stackrel{\mathrm{D}}{=}\left(\sum_{j=1}^{K} A_{j}^{\alpha} W_{j}^{(\alpha)}\right)^{1 / \alpha} Z^{(\alpha)} \\
& \stackrel{\mathrm{D}}{=}\left(W^{(\alpha)}\right)^{1 / \alpha} Z^{(\alpha)} \\
& =X
\end{aligned}
$$

i.e. $X$ is a solution to (4.4).

Example 4.1. (Worst case of FIND.) The limiting distribution of the worst case of the FIND algorithm was characterized in [10] as the unique solution, $S_{0}$, in $M_{2}$ to the fixed-point equation

$$
S \stackrel{\mathrm{D}}{=} U S_{1} \vee(1-U) S_{2}+1,
$$

where $U$ is uniform on [0,1] and $S_{1}$ and $S_{2}$ are i.i.d. copies of $S$ (see also [7]). Moreover, $S_{0}$ has finite moments of any order and an exponentially decreasing tail. In order to study the 
solution set, $\mathcal{L}$, of (4.7), we first note that the class, $\mathcal{W}=\left\{Q_{\lambda}: \lambda \geq 0\right\}$, of Weibull distributions with parameter $\alpha=1$ and distribution functions $F_{\lambda}(x)=\mathrm{e}^{-\lambda / x}, x>0$, with $Q_{0}=\varepsilon_{0}$, are solutions to the homogeneous equation $S \stackrel{\mathrm{D}}{=} U S_{1} \vee(1-U) S_{2}$. Let $X_{\lambda} \stackrel{\mathrm{D}}{=} Q_{\lambda}$. Then, for $\lambda>0$, $X_{\lambda}$ has no finite moments of any order greater than 1 and $\ell_{s}\left(X_{\lambda}, X_{\lambda^{\prime}}\right)=\infty$ for all $\lambda \neq \lambda^{\prime}$ and for $s>1$. The existence theorem (Theorem 4.2) implies that, for all $\lambda \geq 0$, there exists exactly one solution, $S_{\lambda}$, to the worst-case FIND equation (4.7) such that $\ell_{S}\left(X_{\lambda}, S_{\lambda}\right)<\infty$, i.e.

$$
\mathcal{L} \supset\left\{S_{\lambda}: \lambda \geq 0\right\}
$$

Since there are no nonnegative solutions to the related homogeneous additive equation $W \stackrel{\mathrm{D}}{=}$ $U W_{1}+(1-U) W_{2}$, Proposition 4.1 does not add to the set of solutions in this case. It is an open problem as to whether there are further solutions.

Max-recursive stochastic equations arise under quite general conditions as limits of maxrecursive algorithms, as was shown recently in [21]. We finish the paper by restating this limit result as an interesting source of max-recursive equations. We then give an application of this limit theorem to the worst-case behaviour of FIND, characterized by the limiting fixed-point equation stated in (4.7) and discussed above.

Consider a max-recursive sequence $\left(Y_{n}\right)$, with

$$
Y_{n} \stackrel{\mathrm{D}}{=} \bigvee_{r=1}^{K}\left(A_{r}(n) Y_{I_{r}^{(n)}}^{(r)}+b_{r}(n)\right), \quad n \geq n_{0},
$$

induced by an algorithm of divide-and-conquer type. Here $I_{r}^{(n)}$ are subgroup sizes, $b_{r}(n)$ are random toll terms, $A_{r}(n)$ are random weights, and $\left(Y_{n}^{(r)}\right)$ are i.i.d. copies of $\left(Y_{n}\right)$, independent also of $\left(A_{r}(n), b_{r}(n), I^{(n)}\right)$. For a limiting result (after normalization), the following conditions were given in [21]. Assume that the coefficients converge in $L^{s}$ as follows:

$$
\left(A_{1}^{(n)}, \ldots, A_{K}^{(n)}, b_{1}^{(n)}, \ldots, b_{k}^{(n)}\right) \rightarrow_{L^{s}}\left(A_{1}^{*}, \ldots, a_{K}^{*}, b_{1}^{*}, \ldots, b_{K}^{*}\right) .
$$

Then, as a formal limit of (4.8), we obtain

$$
X \stackrel{\mathrm{D}}{=} \bigvee_{r=1}^{K}\left(A_{r}^{*} X_{r}+b_{r}^{*}\right)
$$

as limiting equation. We need the contraction condition for the limit equation, namely

$$
\mathrm{E} \sum_{r=1}^{K}\left|A_{r}^{*}\right|^{s}<1 \quad \text { for some } s>0,
$$

as well as a nondegeneracy condition: for any fixed $\ell$,

$$
\mathrm{E}\left[\mathbf{1}_{\left\{I_{r}^{(n)} \leq \ell\right\} \cup\left\{I_{r}^{(n)}=n\right\}}\left|A_{r}^{(n)}\right|^{s}\right] \rightarrow 0 .
$$

Theorem 4.3. (Max-recursive limit theorem [21].) Let $\left(Y_{n}\right)$ be a max-recursive sequence induced by an algorithm of divide-and-conquer type as in (4.8), and assume that conditions (4.9), (4.11), and (4.12) hold. Then $\ell_{s}\left(Y_{n}, Y^{*}\right) \rightarrow 0$, where $Y^{*}$ is the unique solution to the limit equation (4.10) in $M_{s}$. 
As an application of Theorem 4.3, we next give a direct proof of the limiting worst-case behaviour of FIND. For an alternative stochastic process approach, see [10].

Example 4.2. Let $Y_{n, \ell}$ denote the number of comparisons of the FIND algorithm required for finding the $\ell$ th order statistic. Then

$$
\frac{Y_{n, \ell}}{n} \stackrel{\mathrm{D}}{=} \frac{V-1}{n} \mathbf{1}_{\{V>\ell\}} \frac{Y_{V-1, \ell}}{V-1}+\frac{n-V}{n} \mathbf{1}_{\{V<\ell\}} \frac{\bar{Y}_{n-V, \ell-V}}{n-V-1}+\frac{n-1}{n},
$$

where $V$ is uniform on $\{1, \ldots, n\}$. With $V=\lceil U\rceil$ (i.e. the smallest natural number greater than $U), U$ uniform on $[0,1]$, and the normalization $X_{n, \ell}:=Y_{n, \ell} / n$, we obtain

$$
\begin{aligned}
X_{n, \ell} \stackrel{\mathrm{D}}{=} & \frac{\lceil n U\rceil-1}{n} \mathbf{1}_{\{\lceil n u\rceil / n>\ell / n\}} X_{\lceil n u\rceil-1, \ell} \\
& +\frac{n-\lceil n U\rceil}{n} \mathbf{1}_{\{\lceil n U\rceil / n<\ell / n\}} \bar{X}_{n-\lceil n U\rceil, \ell-\lceil n U\rceil}+\frac{n-1}{n} .
\end{aligned}
$$

Defining the worst case by $M_{n}:=\max _{1 \leq \ell \leq n} X_{n, \ell}$, we obtain the recursive equation

$$
M_{n} \stackrel{\mathrm{D}}{=} \frac{n-1}{n}+\frac{\lceil n U\rceil-1}{n} M_{\lceil n U\rceil-1} \vee \frac{n-\lceil n U\rceil}{n} \bar{M}_{n-\lceil n U\rceil} .
$$

This leads to the limit equation

$$
S \stackrel{\mathrm{D}}{=} 1+U S \vee(1-U) \bar{S}
$$

the worst-case FIND equation. All conditions of Theorem 4.3 are satisfied, for any $s>1$. For any $s>1$, we therefore obtain $\ell_{s}\left(M_{n}, S\right) \rightarrow 0$, where $S$ is the unique solution to (4.13) in $M_{s}$. Thus, uniqueness holds in $\bigcup_{s>1} M_{s}$.

\section{Acknowledgements}

The author would like to thank U. Rösler, for the suggestion to use the weighted branching process construction to establish the existence of solutions via $\ell_{s}$-metrics, and R. Neininger, for some useful discussions on this subject. Thanks are also due to a reviewer for some useful remarks.

\section{References}

[1] Aldous, D. J. and Bandyopadhyay, A. (2005). A survey of max-type recursive distributional equations. Ann. Appl. Prob. 15, 1047-1110.

[2] Alsmeyer, G. And Rösler, U. (2003). The best constant in the Topchii-Vatutin inequality for martingales. Statist. Prob. Lett. 65, 199-206.

[3] Biggins, J. D. (1977). Martingale convergence in the branching random walk. J. Appl. Prob. 14, $25-37$.

[4] Biggins, J. D. And Kyprianou, A. E. (1997). Seneta-Heyde norming in the branching random walk. Ann. Prob. 25, 337-360.

[5] Caliebe, A. And Rösler, U. (2003). Fixed points with finite variance of a smoothing transformation. Stoch. Process. Appl. 107, 105-129.

[6] Cramer, M. And Rüschendorf, L. (1996). Convergence of a branching type recursion. Ann. Inst. H. Poincaré Prob. Statist. 32, 725-741.

[7] Devroye, L. (2001). On the probabilistic worst-case time of 'FIND'. Algorithmica 31, 291-303.

[8] Durrett, R. And Liggett, T. M. (1983). Fixed points of the smoothing transformation. Z. Wahrscheinlichkeitsth. 64, 275-301.

[9] Fill, J. A. And Janson, S. (2000). A characterization of the set of fixed points of the Quicksort transformation. Electron. Commun. Prob. 5, 77-84. 
[10] Grübel, R. AND Rösler, U. (1996). Asymptotic distribution theory for Hoare's selection algorithm. Adv. Appl. Prob. 28, 252-269.

[11] Guivarc'H, Y. (1990). Sur une extension de la notion de loi semi-stable. Ann. Inst. H. Poincaré Prob. Statist. 26, 261-285.

[12] Holley, R. AND Liggett, T. M. (1981). Generalized potlatch and smoothing processes. Z. Wahrscheinlichkeitsth. 55, 165-195.

[13] Hutchinson, J. E. AND Rüschendorf, L. (1998). Random fractal measures via the contraction method. Indiana Univ. Math. J. 47, 471-487.

[14] Hutchinson, J. E. And Rüschendorf, L. (2000). Random fractals and probability metrics. Adv. Appl. Prob. 32, 925-947.

[15] Hutchinson, J. E. AND Rüschendorf, L. (2000). Selfsimilar fractals and selfsimilar random fractals. In Fractal Geometry and Stochastics II, eds C. Brandt et al., Birkhäuser, Basel, pp. 109-124.

[16] Jagers, P. AND Rösler, U. (2004). Stochastic fixed point for the maximum. In Mathematics and Computer Science III, eds M. Drmota et al., Birkhäuser, Basel, pp. 165-195.

[17] LIU, Q. (1998). Fixed points of a generalized smoothing transformation and applications to the branching random walk. Adv. Appl. Prob. 30, 85-112.

[18] Lyons, R., Pemantle, R. and Peres, Y. (1995). Conceptual proofs of $L \log L$ criteria for mean behavior of branching processes. Ann. Prob. 23, 1125-1138.

[19] NeINinger, R. AND RüschendoRF, L. (2004). A general limit theorem for recursive algorithms and combinatorial structures. Ann. Appl. Prob. 14, 378-418.

[20] NeININGER, R. AND RÜSCHENDORF, L. (2004). On the contraction method with degenerate limit equation. Ann. Prob. 32, 2838-2856.

[21] NeININGER, R. AND Rüschendorf, L. (2005). Analysis of algorithms by the contraction method: additive and max-recursive sequences. In Interacting Stochastic Systems, eds J.-D. Deuschel and A. Greven, Springer, Berlin, 435-450.

[22] Rachev, S. T. (1991). Probability Metrics and the Stability of Stochastic Models. John Wiley, Chichester.

[23] RaChev, S. T. AND Rüschendorf, L. (1992). Rate of convergence for sums and maxima and doubly ideal metrics. Theory Prob. Appl. 37, 222-235.

[24] Rachev, S. T. and Rüschendorf, L. (1995). Probability metrics and recursive algorithms. Adv. Appl. Prob. 27, 770-799.

[25] Rösler, U. (1991). A limit theorem for 'Quicksort'. RAIRO Inf. Théoret. Appl. 25, 85-100.

[26] Rösler, U. (1992). A fixed point theorem for distributions. Stoch. Process. Appl. 42, 195-214.

[27] RösLer, U. (2001). On the analysis of stochastic divide and conquer algorithms. Algorithmica 29, $238-261$.

[28] Rösler, U. (2003). Notes on fixed points of max recursive sequences. Preprint.

[29] RöSler, U. ANd RüSChENdORF, L. (2001). The contraction method for recursive algorithms. Algorithmica 29, $3-33$.

[30] Vatutin, V. A. AND Topchĭ, V. A. (1997). The maximum of critical Galton-Watson processes, and leftcontinuous random walks. Theory Prob. Appl. 42, 17-27. 